\title{
Conditional states and entropy in qudit-qubit systems
}

\author{
M. Bilkis,${ }^{1,2}$ N. Canosa, ${ }^{1}$ R. Rossignoli,,${ }^{1,3}$ and N. Gigena ${ }^{1}$ \\ ${ }^{1}$ Instituto de Física de La Plata, CONICET, and Departamento de Física, Universidad Nacional de La Plata, \\ CC 67, La Plata 1900, Argentina \\ ${ }^{2}$ Física Teòrica: Informació i Fenòmens Quàntics, Departament de Física, Universitat Autònoma de Barcelona, \\ 08193 Bellaterra, Barcelona, Spain \\ ${ }^{3}$ Comisión de Investigaciones Científicas de la Provincia de Buenos Aires, La Plata 1900, Argentina
}

(Received 28 December 2018; published 25 June 2019)

\begin{abstract}
We examine, in correlated mixed states of qudit-qubit systems, the set of all conditional qubit states that can be reached after local measurements at the qudit based on rank-1 projectors. While for a similar measurement at the qubit the conditional postmeasurement qudit states lie on the surface of an ellipsoid, for a measurement at the qudit we show that the set of postmeasurement qubit states can form more complex solid regions. In particular, we show the emergence, for some classes of mixed states, of sets which are the convex hull of solid ellipsoids and which may lead to conelike and trianglelike shapes in limit cases. We also analyze the associated measurement-dependent conditional entropy, providing a full analytic determination of its minimum and of the minimizing local measurement at the qudit for the previous states. Separable rank-2 mixtures are also discussed.
\end{abstract}

DOI: 10.1103/PhysRevA.99.062119

\section{INTRODUCTION}

The study of quantum correlations and nonclassical properties in composite quantum systems is of great current interest, having deep implications in the field of quantum information [1-5]. A closely related nontrivial problem is that of the determination of the set of postmeasurement conditional states of one component after a remote local measurement on the other constituents. In this context, the concept of quantum steering ellipsoid [6-14], also known as correlation ellipsoid $[9,14]$, which denotes the set of all Bloch vectors to which one party could collapse if the remote party were able to perform all possible measurements on its side, has provided a useful geometric picture in two-qubit [7-15] and also in multiqubit $[12,16,17]$ systems. Recently, the experimental validation of the quantum steering ellipsoid for different two-qubit states was reported [17].

This geometric approach has been important for understanding the measurement-dependent conditional entropy and its minimizing measurement $[7-9,14,18]$. This entropy measures the average conditional uncertainty in the postmeasurement state of the unmeasured constituent and its minimum plays a key role in the definition of the quantum discord $[2,3,19-22]$. It is also directly related to the entanglement of formation with a purifying third system [23]. The steering ellipsoid has also provided necessary and sufficient conditions for the presence of entanglement in two-qubit systems [10-12], as well as strong monogamy relations determined by its volume in multiqubit states $[12,16,17]$. The set of postmeasurement reduced states in composite systems plays a central role also in the problem of quantum steering [24-30].

Most studies, however, have been concerned with measurements on a qubit component, where the set of all possible measurements can be easily parametrized. In this work we consider instead measurements on a general qudit with dimension $d \geqslant 3$ and analyze, for mixed states of correlated qudit-qubit systems, the set of the ensuing conditional states of the unmeasured qubit after such measurements. We first recall that while in bipartite pure states the conditional state of $B$ after a local measurement at the other system $A$ (based on rank-1 local projectors) is a pure state (which can be any pure state if the original global state is entangled and the reduced state $\rho_{B}$ has full rank), in the case of mixed states the conditional state of the unmeasured system will be in general mixed and lie within a certain subset of the full accessible space, which is essentially determined by the socalled correlation tensor of the global system $[6,9,10,14,18]$ (see the next section). Such a set may include states ranging from pure states to maximally mixed states. In addition, in the case of a qudit-qubit system, if such a measurement is performed on the qubit, the set of conditional states of the unmeasured qudit forms the surface of a three-dimensional ellipsoid [14,18].

Here we will show, however, that for measurements on the qudit, the set of conditional qubit states can form more complex geometries, such as the convex hull of distinct solid ellipsoids and also conelike and trianglelike shapes in limit cases, providing analytic expressions. We will also analyze the associated measurement-dependent conditional entropy, providing general analytic results for its minimizing local measurement at the qudit for certain classes of states, valid for general entropic forms, together with their geometric picture.

We point out that qudit-qubit systems admit several different physical realizations. In particular, it suffices to consider the polarization degrees of freedom of a single photon as the qubit, while the qudit may correspond to its path degrees of freedom. Both can be entangled through the use of beam displacers (as in [17]) or spatial light modulators (SLMs) (see, for instance, [31-33]). Correlated qudit-qudit states can also be realized with two spontaneous parametric down-conversion photons using both polarization and the transverse spatial correlations [34-36]. In addition, for qudits encoded in slit 
states generated through a SLM, general measurements on the qudit can be realized, for instance, with the techniques described in [37]. The present results are then relevant for determining the set of conditional polarization states that can be reached by measurements at the spatial qudits when the whole state is mixed. Of course, realizations of correlated qudit-qubit states through spin chains and arrays are also feasible (see, for example, [38-40]).

The formalism is discussed in Sec. II, where we derive analytic results for the set of conditional qubit states after a local measurement at the qudit for several classes of correlated qudit-qubit states. In Sec. III we examine the associated measurement-determined conditional entropy, providing analytic results for its minimizing measurement in the previous states. We also include results for general rank-2 separable states, with an application to mixtures of aligned two-spin states of arbitrary spin. Conclusions are provided in Sec. IV.

\section{CONDITIONAL STATES IN BIPARTITE SYSTEMS}

\section{A. Formalism}

We first consider a general bipartite system $A+B$, with subsystem dimensions $d_{A}$ and $d_{B}$. We will use orthogonal local operator bases formed by the identity $\mathbb{1}_{S}$ plus $d_{S}^{2}-1$ Hermitian traceless operators $\sigma_{S}$ satisfying

$$
\operatorname{Tr}\left[\sigma_{S \mu} \sigma_{S \nu}\right]=d_{S} \delta_{\mu \nu}, \quad S=A, B
$$

A general mixed state $\rho_{A B}$ can then be written as [41]

$$
\rho_{A B}=\rho_{A} \otimes \rho_{B}+\frac{1}{d_{A} d_{B}} \sum_{\mu, \nu} C_{\mu \nu} \sigma_{A \mu} \otimes \sigma_{B \nu},
$$

where $\rho_{A(B)}=\operatorname{Tr}_{B(A)} \rho_{A B}$ are the reduced states

$$
\rho_{S}=\frac{1}{d_{S}}\left(\mathbb{1}_{S}+\boldsymbol{r}_{S} \cdot \boldsymbol{\sigma}_{S}\right),
$$

with $\boldsymbol{r}_{S}=\left\langle\boldsymbol{\sigma}_{S}\right\rangle=\operatorname{Tr}_{S}\left[\rho_{S} \boldsymbol{\sigma}_{S}\right]$, while

$$
C_{\mu \nu}=\left\langle\sigma_{A \mu} \otimes \sigma_{B \nu}\right\rangle-\left\langle\sigma_{A \mu}\right\rangle\left\langle\sigma_{B \nu}\right\rangle
$$

are the elements of the correlation tensor [14].

We now assume that a local measurement based on rank-1 local projectors is performed on side $A$. It is worth mentioning that this type of positive-operator-valued measure (POVM) is sufficient to minimize the measurement-dependent conditional entropy [2,18]. Moreover, they allow for analytical expressions in the case of simple entropic forms [18] (see Sec. III C for further details). The projectors can be expressed as

$$
\Pi_{\boldsymbol{k}}^{A}=\left|\boldsymbol{k}_{A}\right\rangle\left\langle\boldsymbol{k}_{A}\right|=\frac{1}{d_{A}}\left(\mathbb{1}_{A}+\boldsymbol{k} \cdot \boldsymbol{\sigma}_{A}\right),
$$

with $\boldsymbol{k}=\left\langle\boldsymbol{k}_{A}\left|\boldsymbol{\sigma}^{A}\right| \boldsymbol{k}_{A}\right\rangle$ a vector satisfying $|\boldsymbol{k}|^{2}=d_{A}-1$. The conditional postmeasurement state of $B$ is

$$
\begin{aligned}
\rho_{B / \boldsymbol{k}} & =p_{\boldsymbol{k}}^{-1} \operatorname{Tr}_{A}\left[\rho_{A B} \Pi_{\boldsymbol{k}}^{A} \otimes \mathbb{1}_{B}\right]=p_{\boldsymbol{k}}^{-1}\left\langle\boldsymbol{k}_{A}\left|\rho_{A B}\right| \boldsymbol{k}_{A}\right\rangle \\
& =\frac{1}{d_{B}}\left[\mathbb{1}+\boldsymbol{r}_{B / \boldsymbol{k}} \cdot \boldsymbol{\sigma}_{B}\right],
\end{aligned}
$$

where $p_{\boldsymbol{k}}=\operatorname{Tr}_{A}\left[\rho_{A} \Pi_{\boldsymbol{k}}^{A}\right]=\frac{1}{d_{A}}\left(1+\boldsymbol{r}_{A} \cdot \boldsymbol{k}\right)$ is the probability of measuring state $\left|\boldsymbol{k}_{A}\right\rangle$ and

$$
\boldsymbol{r}_{B / \boldsymbol{k}}=\operatorname{Tr}_{B}\left[\rho_{B / \boldsymbol{k}} \boldsymbol{\sigma}_{B}\right]=\boldsymbol{r}_{B}+\frac{C^{T} \boldsymbol{k}}{1+\boldsymbol{r}_{A} \cdot \boldsymbol{k}}
$$

is the conditional average of $\boldsymbol{\sigma}_{B}$ after result $\boldsymbol{k}$ at $A$.

The complete local measurement will be defined by a set of $m$ operators $M_{j}^{A}=\sqrt{r_{j}} \Pi_{\boldsymbol{k}_{j}}^{A}, r_{j}>0$, satisfying $\sum_{j}\left(M_{j}^{A}\right)^{\dagger} M_{j}^{A}=\sum_{j} r_{j} \Pi_{\boldsymbol{k}_{j}}^{A}=\mathbb{1}_{A}$, i.e.,

$$
\sum_{j} r_{j}=d_{A}, \quad \sum_{j} r_{j} \boldsymbol{k}_{j}=\mathbf{0} .
$$

The probability of result $j$ is $p_{j}=r_{j} p_{\boldsymbol{k}_{j}}$, with (8) ensuring $\sum_{j} p_{j}=1$ and $\sum_{j} p_{j} \boldsymbol{r}_{B / \boldsymbol{k}_{j}}=\boldsymbol{r}_{B}$, i.e., $\sum_{j} p_{j} \rho_{B / \boldsymbol{k}_{j}}=\rho_{B}$, preventing faster than light signaling from $A$ to $B$. Standard von Neumann measurements correspond to $m=d_{A}, r_{j}=1 \forall j$, and $\Pi_{\boldsymbol{k}_{j}}^{A}$ orthogonal projectors.

If $\rho_{A B}$ has local support on a certain subspace $\mathcal{S}_{A}$ of $A$ of dimension $d_{A}^{\prime}<d_{A}$, we can always write

$$
\left|\boldsymbol{k}_{A}\right\rangle=\sqrt{q}\left|\boldsymbol{k}_{A}^{\|}\right\rangle+\sqrt{1-q}\left|\boldsymbol{k}_{A}^{\perp}\right\rangle,
$$

where $\left|\boldsymbol{k}_{A}^{\|}\right\rangle \in \mathcal{S}_{A}, q \in[0,1]$, and $\left|\boldsymbol{k}_{A}^{\perp}\right\rangle$ is orthogonal to $\mathcal{S}_{A}$ such that $\left\langle\boldsymbol{k}_{A}\left|\rho_{A B}\right| \boldsymbol{k}_{A}\right\rangle=q\left\langle\boldsymbol{k}_{A}^{\|}\left|\rho_{A B}\right| \boldsymbol{k}_{A}^{\|}\right\rangle$. Hence, for conditional states the effects of a complete local measurement based on operators $M_{j}^{A}=\sqrt{r_{j}} \Pi_{\boldsymbol{k}_{j}}^{A}$ are the same as those of a measurement in the subspace $\mathcal{S}_{A}$ based on operators $M_{j}^{\prime}=\sqrt{q_{j} r_{j}} \Pi_{\boldsymbol{k}_{j}^{\| \prime}}^{A}$, satisfying $\sum_{j} M_{j}^{\prime \dagger} M_{j}^{\prime}=\mathbb{1}_{d_{A}^{\prime}}$.

In what follows we will consider a qudit-qubit system $\left(d_{B}=2\right)$. We will analyze the whole set of postmeasurement conditional qubit states $\rho_{B / \boldsymbol{k}}$ of $B$, characterized by the now Bloch vectors (7) ( $\sigma_{B}$ are now the standard Pauli operators), that can be reached for any possible rank-1 projector $\Pi_{k}^{A}$. We first recall that for similar measurements at the qubit, the set of all conditional postmeasurement vectors $\boldsymbol{r}_{A / \boldsymbol{k}}$ of qudit $A$ forms the surface of a three-dimensional ellipsoid (for a rank-3 correlation tensor) [6,14], whose semiaxes are determined by the correlation tensor $C$ and the vector $\boldsymbol{r}_{B}$. In contrast, for a measurement on the qudit $A$, we will show that the set of all postmeasurement qubit Bloch vectors $\boldsymbol{r}_{B / \boldsymbol{k}}$ will be in general a region with finite volume, which may have shapes more general than a single ellipsoid.

\section{B. Mixture of a pure state with the maximally mixed state}

As a first example, we consider the qudit-qubit state

$$
\rho_{A B}=p|\Psi\rangle\langle\Psi|+(1-p) \frac{\mathbb{1}_{A B}}{2 d_{A}},
$$

where $|\Psi\rangle \equiv\left|\Psi_{A B}\right\rangle$ stands for a general pure state and $p \in$ $[0,1]$. Positivity of $\rho_{A B}$ is nonetheless ensured for $-\frac{1}{2 d_{A}-1} \leqslant$ $p \leqslant 1$, with negative values of $p$ representing depletion of state $|\Psi\rangle$ from the maximally mixed state.

By means of the Schmidt decomposition of $|\Psi\rangle$, we may always choose orthogonal local states $\left|0_{A(B)}\right\rangle$ and $\left|1_{A(B)}\right\rangle$ such that $|\Psi\rangle$ can be written as

$$
|\Psi\rangle=\cos \frac{\beta}{2}|00\rangle+\sin \frac{\beta}{2}|11\rangle,
$$




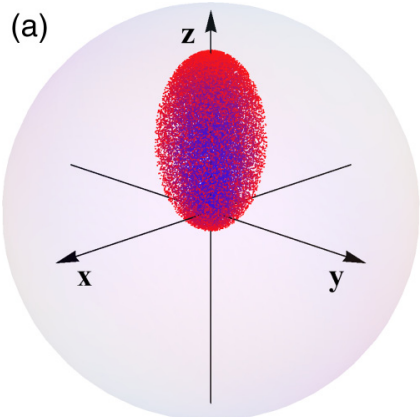

(b)

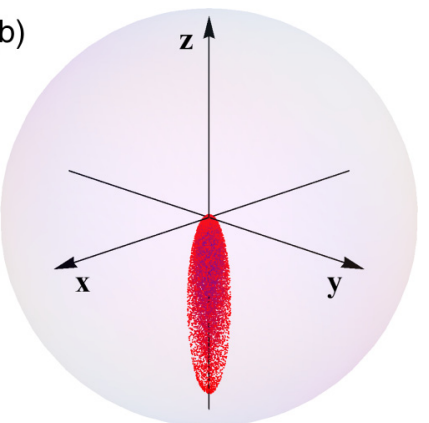

FIG. 1. (a) Set of conditional postmeasurement states of qubit $B$, represented by the Bloch vectors (14) within the qubit Bloch sphere, after random measurements on qudit $A$ based on rank-1 projectors, for the qudit-qubit mixed state (10) with $p=0.5, \beta=\pi / 10$, and $d_{A}=4$ (for which $\rho_{A B}$ is entangled). The set forms a filled ellipsoid if $p<1$, with the origin at one of its foci and eccentricity determined by $p$ and the entanglement of $|\Psi\rangle$ [Eq. (16)], becoming a sphere when maximally entangled. The color indicates the value of $q$ in (12)-(14) [red (blue) for high (low) $q$ ], which determines the weight of the pure term in the postmeasurement qubit state (13). (b) Ellipsoid with opposite orientation obtained for feasible $\left(p \geqslant-\frac{1}{2 d_{A}-1}\right)$ negative values of $p$ in (10), here shown for $p=-0.14$.

where $|i j\rangle=\left|i_{A}\right\rangle \otimes\left|j_{B}\right\rangle$ and the angle $\beta \in[0, \pi / 2]$ is determined by its concurrence [42] $(|\Psi\rangle$ can be seen as an effective two-qubit state) $C_{A B}=\sin \beta$. The mixed state (10) has then a nonpositive partial transpose $[43,44]$ (i.e., positive negativity [45]) for $|p| \sin \beta>(1-p) / d_{A}$, i.e., $p>\left(1+d_{A} \sin \beta\right)^{-1}>0$.

We now show that for $p<1$ and $d_{A} \geqslant 3$, the set of all possible conditional qubit states $\rho_{B / \boldsymbol{k}}$ after a projective measurement at the qudit with result $\boldsymbol{k}$ will be a filled ellipsoid symmetric around the local $z$ axis, with the origin as one of its foci and the major semiaxis along $z$ [Fig. 1(a)]. The local $z$ axes are of course those defined by the Schmidt states such that $\sigma_{S z}=\left|0_{S}\right\rangle\left\langle 0_{S}|-| 1_{S}\right\rangle\left\langle 1_{S}\right|$.

Proof. We first note that if the state $\left|\boldsymbol{k}_{A}\right\rangle$ of $\Pi_{\boldsymbol{k}}^{A}$ is restricted to the subspace spanned by the states $\left\{\left|0_{A}\right\rangle,\left|1_{A}\right\rangle\right\}$, the situation is similar to that of a two-qubit system and the surface of an ellipsoid will be obtained [14]. However, if $\left|\boldsymbol{k}_{A}\right\rangle$ has just a component within this subspace, $\boldsymbol{r}_{B / \boldsymbol{k}}$ will lie within the previous ellipsoid due to the smaller overlap with the state $|\Psi\rangle$, filling the whole ellipsoid as this component diminishes. We now prove this result explicitly, providing the ellipsoid parameters. Discarding a global phase, a general pure qudit state $\left|\boldsymbol{k}_{A}\right\rangle$ can be here written as [Eq. (9)]

$$
\begin{aligned}
\left|\boldsymbol{k}_{A}\right\rangle & =\sqrt{q}\left|\boldsymbol{k}_{A}^{\|}\right\rangle+\sqrt{1-q}\left|\boldsymbol{k}_{A}^{\perp}\right\rangle, \\
\left|\boldsymbol{k}_{A}^{\|}\right\rangle & =\cos \frac{\alpha}{2}\left|0_{A}\right\rangle+\sin \frac{\alpha}{2} e^{-i \phi}\left|1_{A}\right\rangle,
\end{aligned}
$$

with $q \in[0,1], \alpha \in[0, \pi], \phi \in[0,2 \pi)$, and $\left|\boldsymbol{k}_{A}^{\perp}\right\rangle$ orthogonal to $\left|0_{A}\right\rangle$ and $\left|1_{A}\right\rangle$. The normalized conditional postmeasurement qubit state (6) becomes

$$
\rho_{B / \boldsymbol{k}}=p_{\boldsymbol{k}}^{-1}\left[p q\left\langle\boldsymbol{k}_{A}^{\|} \mid \Psi\right\rangle\left\langle\Psi \mid \boldsymbol{k}_{A}^{\|}\right\rangle+\frac{1-p_{1}}{2 d_{A}} \mathbb{1}_{B}\right],
$$

with $\left\langle\boldsymbol{k}_{A}^{\|} \mid \Psi\right\rangle=\cos \frac{\alpha}{2} \cos \frac{\beta}{2}\left|0_{B}\right\rangle+e^{i \phi} \sin \frac{\alpha}{2} \sin \frac{\beta}{2}\left|1_{B}\right\rangle \quad$ and $p_{k}=\frac{1}{2} p q(1+\cos \beta \cos \alpha)+\frac{1-p}{d_{A}}$.

The ensuing Bloch vector $\boldsymbol{r}_{B / \boldsymbol{k}}=\operatorname{Tr}\left[\rho_{B / \boldsymbol{k}} \boldsymbol{\sigma}\right]$ is

$\boldsymbol{r}_{B / \boldsymbol{k}}=\frac{p q}{2 p_{\boldsymbol{k}}}(\sin \alpha \sin \beta \cos \phi, \sin \alpha \sin \beta \sin \phi, \cos \alpha+\cos \beta)$

$$
=r_{B / k}(\sin \theta \cos \phi, \sin \theta \sin \phi, \cos \theta),
$$

where (14) is its polar representation, with $\cos \theta=$ $\frac{\cos \alpha+\cos \beta}{1+\cos \alpha \cos \beta}$, and $r_{B / k}$ is given by

$$
r_{B / k}=\frac{a\left(1-e^{2}\right)}{1-e \cos \theta},
$$

with $\theta \in[0, \pi]$ if $\alpha \in[0, \pi]$ (and $\beta \neq 0$ ). Here

$$
\begin{aligned}
& e=\frac{(1-p) \cos \beta}{1-p+\frac{1}{2} p q d_{A} \sin ^{2} \beta}, \\
& a=\frac{p q\left(\frac{1-p}{d_{A}}+\frac{1}{2} p q \sin ^{2} \beta\right)}{2 \Delta},
\end{aligned}
$$

with $\Delta=\left(\frac{1-p}{d_{A}}+p q \sin ^{2} \frac{\beta}{2}\right)\left(\frac{1-p}{d_{A}}+p q \cos ^{2} \frac{\beta}{2}\right)$.

Thus, at fixed $\beta>0$ and $q>0$, Eq. (15) represents an ellipsoid symmetric around the $z$ axis with eccentricity $|e|$ and major semiaxis of length $|a|$ along $z$, with the origin at its focus. All ellipsoids are enclosed within that for $q=1$, for which $|a|$ is maximum. Thus, for $d_{A} \geqslant 3$, variation of $q$ in the interval $[0,1]$ leads to a filled ellipsoid, as shown in Fig. 1. In the qubit case $d_{A}=2, q=1$, and just its surface remains.

In Cartesian coordinates, the ellipsoid equation reads

$$
\frac{x^{2}+y^{2}}{b^{2}}+\frac{\left(z-z_{c}\right)^{2}}{a^{2}}=1,
$$

where $b=a \sqrt{1-e^{2}}=p q \sin \beta / 2 \sqrt{\Delta}$ is the minor semiaxis length and $z_{c}=a e=p q \frac{1-p}{2 d_{A} \Delta} \cos \beta$ the $z$ coordinate of its center. Let us now verify some limit cases. For $\beta=\pi / 2$, $|\Psi\rangle$ is maximally entangled and $e=0$ : The filled ellipsoid becomes a filled sphere of radius $a=b=\left[1+\frac{2(1-p)}{p d_{A}}\right]^{-1}$, centered at the origin $\left(z_{c}=0\right)$. On the other hand, for $\beta=0$, $|\Psi\rangle$ becomes a product state and $e=1$, implying that the ellipsoid reduces to a segment along the $z$ axis $(b=0$ and $\left.z_{c}=a\right)$, starting at the origin and ending at $2 a=\left[1+\frac{1-p}{p q d_{A}}\right]^{-1}$.

Finally, in the pure state limit $p=1$, the set of postmeasurement states becomes the whole Bloch sphere surface (it is always pure) for any entangled $|\Psi\rangle$, as $e \rightarrow 0$ and $a \rightarrow 1$ for any $\beta \in(0, \pi)$, while for a separable $|\Psi\rangle(\beta=0)$ it obviously reduces to the point $(1,0,0)=\boldsymbol{r}_{B}$. If the qudit dimension $d_{A}$ increases [at fixed $\beta$ and $p \in(0,1)], a$ and hence the volume of the ellipsoid increases, whereas the eccentricity decreases. For $p q d_{A} \gg 1, a \approx 1-\frac{2(1-p)}{p q d_{A} \sin ^{2} \beta}$ and $e \approx \frac{2(1-p) \cos \beta}{p q d_{A} \sin ^{2} \beta}$.

We also note that Eqs. (15)-(17) remain valid for negative values $\frac{-1}{2 d_{A}-1} \leqslant p<0$, in which case $a$ and $z_{c}$ change sign. Hence, an ellipsoid also follows from a state $\rho_{A B}$ maximally mixed in the $\left(2 d_{A}-1\right)$-dimensional subspace orthogonal to $|\Psi\rangle$ [Fig. 1(b)].

\section{Mixture of two pure states with the maximally mixed state}

For other states $\rho_{A B}$, the set of postmeasurement qubit states may adopt more complex forms. Let us consider, for 


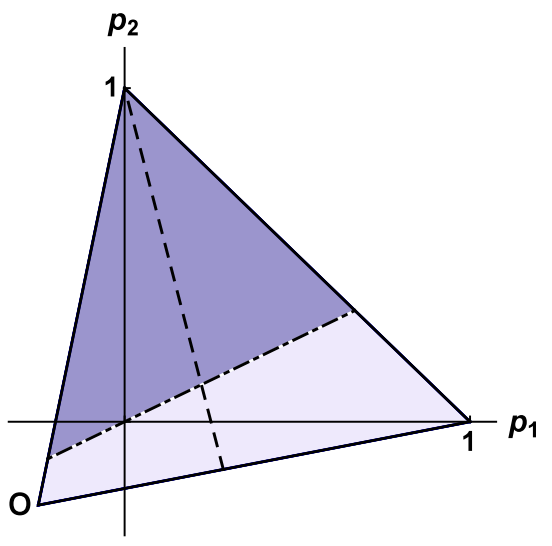

FIG. 2. Triangle in $\left(p_{1}, p_{2}\right)$ space corresponding to a physical state in the qudit-qubit mixed state (19). Here $O$ indicates the vertex at $\frac{-1}{2 d_{A}-2}(1,1)$. In the case $\beta_{1}=\pi / 2$ and $\beta_{2}=0$, points $\left(p_{1}, p_{2}\right)$ on the right of the dashed line correspond to an entangled state with nonzero negativity, while those above (below) the dash-dotted line, i.e., dark (light) colored sectors, lead to a filled ice-cream (ellipsoid) shape of the set of postmeasurement qubit states [see Eq. (37)].

instance, the state

$$
\rho_{A B}=p_{1}\left|\Psi_{1}\right\rangle\left\langle\Psi_{1}\left|+p_{2}\right| \Psi_{2}\right\rangle\left\langle\Psi_{2}\right|+p_{0} \frac{\mathbb{1}_{A B}}{2 d_{A}},
$$

where $p_{0}=1-p_{1}-p_{2}$. The condition $\rho_{A B} \geqslant 0$ implies $p_{1}+p_{2} \leqslant 1, \quad p_{1}-\left(2 d_{A}-1\right) p_{2} \leqslant 1$, and $p_{2}-\left(2 d_{A}-1\right) p_{1} \leqslant 1$, which delimit a triangle in the $\left(p_{1}, p_{2}\right)$ plane with vertices at $\left(p_{1}, p_{2}\right)=(1,0),(0,1)$, and $\frac{-1}{2 d_{A}-2}(1,1)$ (Fig. 2). We will focus on the case where the states $\left|\Psi_{1}\right\rangle$ and $\left|\Psi_{2}\right\rangle$ are orthogonal and have orthogonal supports at the qudit side.

\section{Two entangled states}

We first consider the case where these states are entangled such that their Schmidt decompositions are

$$
\begin{aligned}
& \left|\Psi_{1}\right\rangle=\cos \frac{\beta_{1}}{2}\left|00_{1}\right\rangle+\sin \frac{\beta_{1}}{2}\left|11_{1}\right\rangle, \\
& \left|\Psi_{2}\right\rangle=\cos \frac{\beta_{2}}{2}\left|20_{2}\right\rangle+\sin \frac{\beta_{2}}{2}\left|31_{2}\right\rangle,
\end{aligned}
$$

where qudit states $\left|i_{A}\right\rangle, i=0,1,2,3$, are all orthogonal while qubit states $\left|j_{1_{B}}\right\rangle$ are not necessarily orthogonal to $\left|j_{2_{B}}^{\prime}\right\rangle$. We are assuming here $d_{A} \geqslant 4$. The partial transpose will have a negative eigenvalue associated with state $\left|\Psi_{i}\right\rangle$ if $p_{i} \sin \beta_{i}>$ $p_{0} / d_{A}$, i.e., $p_{1}>\frac{1-p_{2}}{1+d_{A} \sin \beta_{1}}$ for $\left|\Psi_{1}\right\rangle$ and $p_{2}>\frac{1-p_{1}}{1+d_{A} \sin \beta_{2}}$ for $\left|\Psi_{2}\right\rangle$, having at most two negative eigenvalues.

The set of reachable postmeasurement qubit states for measurements based on rank-1 local projectors at the qudit will be essentially the convex hull of the ellipsoids associated with each state, as shown in Fig. 3. A general pure state $\left|\boldsymbol{k}_{A}\right\rangle$ of the qudit can now be written as

$$
\left|\boldsymbol{k}_{A}\right\rangle=\sqrt{q^{\prime}}\left(\sqrt{q}\left|\boldsymbol{k}_{1 A}\right\rangle+e^{-i \psi} \sqrt{1-q}\left|\boldsymbol{k}_{2 A}\right\rangle\right)+\sqrt{1-q^{\prime}}\left|\boldsymbol{k}_{A}^{\perp}\right\rangle,
$$

(a)

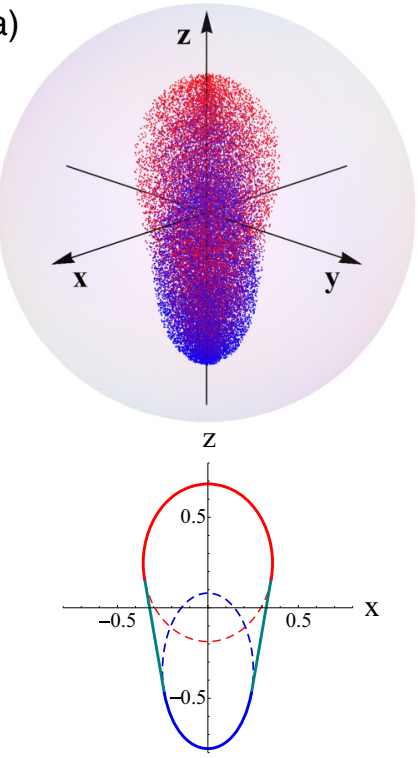

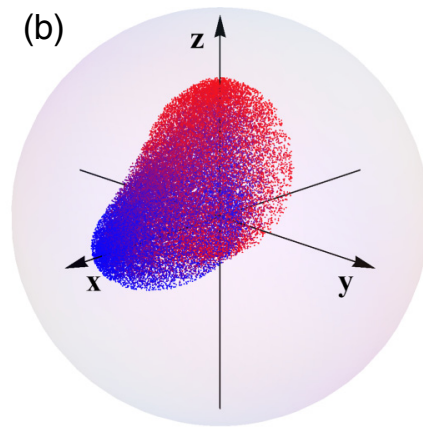

(b)

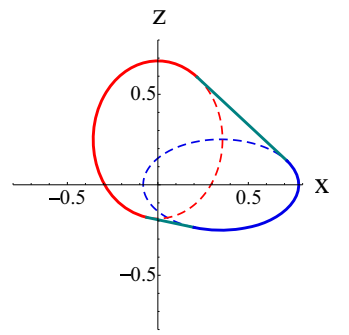

FIG. 3. Set of postmeasurement qubit states after random measurements on the qudit for the state (19). It is the convex hull of the ellipsoids (30) associated with each state $\left|\Psi_{i}\right\rangle$ of the mixture, as indicated in the lower panels, which depict the set boundaries in the $x z$ plane (solid lines), together with the ellipsoids projection (solid and dashed lines). The parameters are $p_{1}=0.2, \beta_{1}=\pi / 5$, $p_{2}=0.3, \beta_{2}=\pi / 10$, and $d_{A}=6$, for which $\rho_{A B}$ is entangled, with the major semiaxes of the ellipsoids in (a) opposite and (b) orthogonal directions. The color indicates the relative weight of each state $\left|\Psi_{i}\right\rangle$ in the final state (24) [the value of $q$ in Eq. (22)], with red (blue) denoting prominence of $\left|\Psi_{1}\right\rangle\left(\left|\Psi_{2}\right\rangle\right)$.

where $q, q^{\prime} \in[0,1],\left|\boldsymbol{k}_{A}^{\perp}\right\rangle$ is orthogonal to all $\left|i_{A}\right\rangle$ with $i \leqslant 3$ (if $d_{A} \leqslant 4$ we set $q^{\prime}=1$ ), and

$$
\begin{aligned}
& \left|\boldsymbol{k}_{1 A}\right\rangle=\cos \frac{\alpha_{1}}{2}\left|0_{A}\right\rangle+e^{-i \phi_{1}} \sin \frac{\alpha_{1}}{2}\left|1_{A}\right\rangle, \\
& \left|\boldsymbol{k}_{2 A}\right\rangle=\cos \frac{\alpha_{2}}{2}\left|2_{A}\right\rangle+e^{-i \phi_{2}} \sin \frac{\alpha_{2}}{2}\left|3_{A}\right\rangle .
\end{aligned}
$$

The resulting conditional state of the qubit is

$$
\begin{aligned}
\rho_{B / \boldsymbol{k}}= & p_{\boldsymbol{k}}^{-1}\left\{q ^ { \prime } \left[q p_{1}\left\langle\boldsymbol{k}_{1 A} \mid \Psi_{1}\right\rangle\left\langle\Psi_{1} \mid \boldsymbol{k}_{1 A}\right\rangle\right.\right. \\
& \left.\left.+(1-q) p_{2}\left\langle\boldsymbol{k}_{2 A} \mid \Psi_{2}\right\rangle\left\langle\Psi_{2} \mid \boldsymbol{k}_{2 A}\right\rangle\right]+\frac{p_{0}}{2 d_{A}} \mathbb{1}_{B}\right\} \\
= & \frac{q p_{\boldsymbol{k}_{1}}}{p_{\boldsymbol{k}}} \rho_{B / \boldsymbol{k}_{1}}+\frac{(1-q) p_{\boldsymbol{k}_{2}}}{p_{\boldsymbol{k}}} \rho_{B / \boldsymbol{k}_{2}},
\end{aligned}
$$

where $\quad\left\langle\boldsymbol{k}_{i A} \mid \Psi_{i}\right\rangle=\cos \frac{\alpha_{i}}{2} \cos \frac{\beta_{i}}{2}\left|0_{i}\right\rangle+e^{i \phi_{i}} \sin \frac{\alpha_{i}}{2} \sin \frac{\beta_{i}}{2}\left|1_{i}\right\rangle$, $p_{k}=q p_{k_{1}}+(1-q) p_{\boldsymbol{k}_{2}}$, and

$$
\begin{aligned}
\rho_{B / \boldsymbol{k}_{i}} & =\frac{1}{p_{\boldsymbol{k}_{i}}}\left(q^{\prime} p_{i}\left\langle\boldsymbol{k}_{i A} \mid \Psi_{i}\right\rangle\left\langle\Psi_{i} \mid \boldsymbol{k}_{i A}\right\rangle+\frac{p_{0}}{2 d_{A}} \mathbb{1}_{B}\right), \\
p_{\boldsymbol{k}_{i}} & =q^{\prime} p_{i}\left(1+\cos \beta_{i} \cos \alpha_{i}\right) / 2+p_{0} / d_{A}
\end{aligned}
$$

are the conditional postmeasurement qubit states and probabilities obtained for $q=1(i=1)$ and $0(i=2)$.

Hence, the postmeasurement state (25) is just the convex combination of the states (26), with $q p_{k_{1}} / p_{\boldsymbol{k}}$ covering all values between 0 and 1 as $q$ varies from 0 to 1 (even for 
negative values of $p_{i}$ provided $\rho_{A B}$ is positive and $q^{\prime} p_{1} p_{2} \neq 0$ ). The Bloch vector obtained from (24) can then be written as

$$
\boldsymbol{r}_{B / \boldsymbol{k}}=\frac{q p_{\boldsymbol{k}_{1}}}{p_{\boldsymbol{k}}} \boldsymbol{r}_{B / \boldsymbol{k}_{1}}+\frac{(1-q) p_{\boldsymbol{k}_{2}}}{p_{\boldsymbol{k}}} \boldsymbol{r}_{B / \boldsymbol{k}_{2}}
$$

where $\boldsymbol{r}_{B / \boldsymbol{k}_{i}}$ are the vectors determined by $\rho_{B / \boldsymbol{k}_{i}}$ :

$$
\begin{aligned}
\boldsymbol{r}_{B / \boldsymbol{k}_{i}}= & \frac{q^{\prime} p_{i}}{2 p_{\boldsymbol{k}_{i}}} R_{i}\left(\sin \alpha_{i} \sin \beta_{i} \cos \phi_{i}, \sin \alpha_{i} \sin \beta_{i} \sin \phi_{i},\right. \\
& \left.\cos \alpha_{i}+\cos \beta_{i}\right) \\
= & r_{B / \boldsymbol{k}_{i}} R_{i}\left(\sin \theta_{i} \cos \phi_{i}, \sin \theta_{i} \sin \phi_{i}, \cos \theta_{i}\right) .
\end{aligned}
$$

Here $R_{i}$ are the operators rotating the original $z$ axis to the $z_{i}$ axis determined by the states $\left|0_{i}\right\rangle$ and $\left|1_{i}\right\rangle$, while $\cos \theta_{i}=\frac{\cos \alpha_{i}+\cos \beta_{i}}{1+\cos \alpha_{i} \cos \beta_{i}}$ and

$$
r_{B / k_{i}}=\frac{a_{i}\left(1-e_{i}^{2}\right)}{1-e_{i} \cos \theta_{i}},
$$

with

$$
\begin{aligned}
e_{i} & =\frac{p_{0} \cos \beta_{i}}{p_{0}+\frac{1}{2} p_{i} q^{\prime} d_{A} \sin ^{2} \beta_{i}}, \\
a_{i} & =\frac{p_{i} q^{\prime}\left(p_{0} / d_{A}+\frac{1}{2} p_{i} q^{\prime} \sin ^{2} \beta_{i}\right)}{2\left(p_{0} / d_{A}+p_{i} q^{\prime} \cos ^{2} \frac{\beta_{i}}{2}\right)\left(p_{0} / d_{A}+p_{i} q^{\prime} \sin ^{2} \frac{\beta_{i}}{2}\right)} .
\end{aligned}
$$

Therefore, the ensuing set of postmeasurement vectors obtained for all values of $\alpha_{1}, \phi_{1}, \alpha_{2}, \phi_{2}, q$, and $q^{\prime}$ in (22) and (23) will be the convex hull of the filled ellipsoids (29) determined by $\boldsymbol{r}_{B / \boldsymbol{k}_{i}}$. All sets will be contained within that obtained for $q^{\prime}=1$, entailing that the set can be obtained by setting $q^{\prime}=1$ (and varying all other measurement parameters). The same set is then also obtained for $d=4$ (where $q^{\prime}=1$ ). The present results can be straightforwardly extended to a mixture of several pure states $\left|\Psi_{i}\right\rangle$ with orthogonal supports at $A$.

As illustration, Fig. 4 depicts the resulting figure when both ellipsoids have collinear [Fig. 4(a)] or orthogonal [Fig. 4(b)] major semiaxes, for $d_{A}=6$. We note that the ensuing $\rho_{A B}$ is entangled, with two negative eigenvalues of the partial transpose. The condition which ensures that the major semiaxes of the second ellipsoid will protrude above the first ellipsoid surface is just

$$
p_{2} \geqslant \frac{p_{1} \sin ^{2} \beta_{1}}{\left(1+\cos \beta_{2}\right)\left(1-\cos \beta_{1} \cos \gamma\right)},
$$

where $\gamma$ is the angle between both major semiaxes and we have assumed $p_{i} \geqslant 0$ (for negative values the inequality should be inverted).

\section{Limit cases}

a. Ice-cream shapes. We now examine the case where one of the states $\left|\Psi_{i}\right\rangle$ is separable. In this case we can consider $d_{A} \geqslant 3$. When $\beta_{2}=0,\left|\Psi_{2}\right\rangle$ in (19) becomes separable,

$$
\left|\Psi_{2}\right\rangle=\left|2_{A}\right\rangle\left|0_{2}\right\rangle, \quad\left|0_{2}\right\rangle=\cos \frac{\gamma}{2}\left|0_{1}\right\rangle+e^{i \eta} \sin \frac{\gamma}{2}\left|1_{1}\right\rangle,
$$

(a)
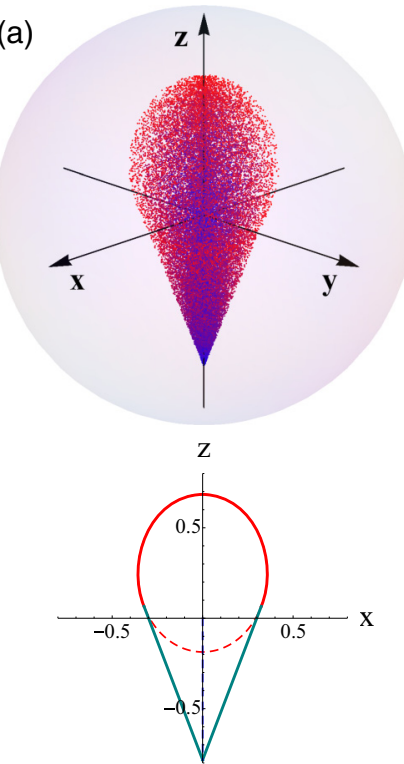

(b)
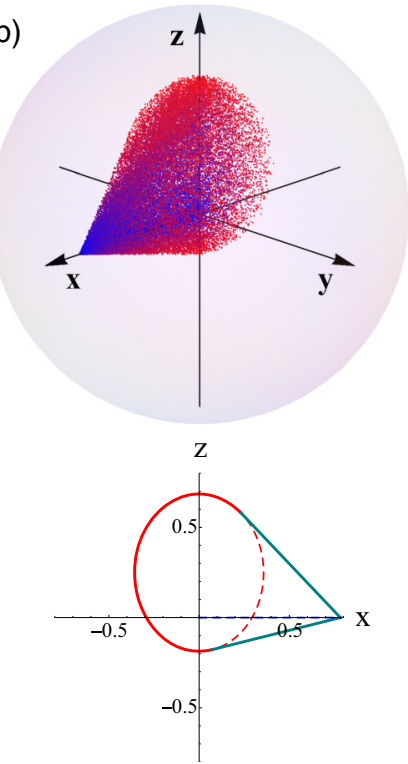

FIG. 4. Set of postmeasurement qubit states after random measurements on the qudit, for the mixture (19) with an entangled state $\left|\Psi_{1}\right\rangle\left(\beta_{1}=\pi / 5\right)$ and a separable state $\left|\Psi_{2}\right\rangle\left(\beta_{2}=0\right)$. The resulting set is a cone topped with an ellipsoid (ice-cream shape), with the cone vertex $r_{v}$ determined by the separable state $\left|\Psi_{2}\right\rangle$ [Eq. (36)]. The plots correspond to $r_{v}$ in the (a) $-z$ axis and (b) $+x$ axis, with $p_{1}=0.2$, $p_{2}=0.3$, and $d_{A}=6$. The lower panels depict the formation of the cone as a result of the convex hull of the ellipsoid and the point $\boldsymbol{r}_{v}$.

where $\left|0_{2}\right\rangle$ is an arbitrary qubit state. The second ellipsoid then reduces to a segment: We obtain

$$
\boldsymbol{r}_{B / \boldsymbol{k}_{2}}=\frac{q^{\prime} p_{2} \cos ^{2} \frac{\alpha_{2}}{2}}{q^{\prime} p_{2} \cos ^{2} \frac{\alpha_{2}}{2}+p_{0} / d_{A}}\left\langle 0_{2}|\boldsymbol{\sigma}| 0_{2}\right\rangle,
$$

with $\left\langle 0_{2}|\sigma| 0_{2}\right\rangle=(\sin \gamma \cos \eta, \sin \gamma \sin \eta, \cos \gamma)$. Therefore, varying $q^{\prime}$ and/or $\alpha_{2}$ leads to a segment linking the origin with the vector

$$
\boldsymbol{r}_{v}=\frac{p_{2}(\sin \gamma \cos \eta, \sin \gamma \sin \eta, \cos \gamma)}{p_{2}+p_{0} / d_{A}} .
$$

By conveniently choosing the $x$ axis we may obviously always set $\eta=0$. In the qutrit case $d_{A}=3, q^{\prime}=1$, and $\alpha_{2}=0$, so here $\boldsymbol{r}_{B / \boldsymbol{k}_{2}}=\boldsymbol{r}_{v}$.

The final set obtained after covering all values of $q \in[0,1]$ and $\alpha_{1}$ and $\phi_{1}$ will lead to the convex hull of the first ellipsoid and the segment ending in $\boldsymbol{r}_{v}$ (or equivalently the point $\boldsymbol{r}_{v}$ ). The parameter $q^{\prime}$ will have no effect in the full final set, since the variation of $q$ will already produce a filled volume, so this result is also valid for $d_{A}=3$.

If $\boldsymbol{r}_{v}$ lies within the ellipsoid, the final set will still be a filled ellipsoid. However, when it lies outside, the final figure will be a cone with vertex at $\boldsymbol{r}_{v}$ topped with the ellipsoid, with the cone straight borders ending tangent to the ellipsoid surface. This leads to an ice-cream-like shape, as seen in Fig. 4 for the cases where the segment is collinear [Fig. 4(a)] or orthogonal [Fig. 4(b)] to the major semiaxis of the ellipsoid. 


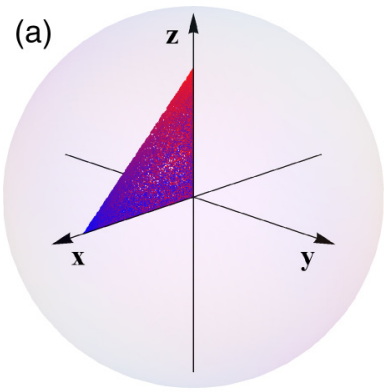

(b)

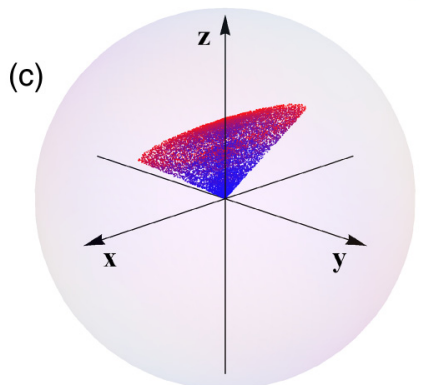

FIG. 5. Set of postmeasurement qubit states after random measurements on the qudit, for the mixture (19) with both $\left|\Psi_{1}\right\rangle$ and $\left|\Psi_{2}\right\rangle$ separable $\left(\beta_{1}=\beta_{2}=0\right)$. (a) For $p_{1}+p_{2}<1$ and $d_{A} \geqslant 3$, it is a two-dimensional triangle [if $\gamma \in(0, \pi)$ in (34)], as shown for $p_{1}=0.3, p_{2}=0.2, \gamma=\pi / 2, \eta=0$, and $d_{A}=4$. (b) When $p_{1}+$ $p_{2}=1$, the triangle reduces to the segment joining the pure states Bloch vectors. Such needlelike shape holds for any mixture of two arbitrary pure separable states like Eqs. (38)-(39), and any $d_{A} \geqslant 2$. (c) On the other hand, for $p_{1}+p_{2}<1, d_{A} \geqslant 3$, and $\left|\Psi_{1,2}\right\rangle$ separable but with nonorthogonal supports at the qudit side, the set becomes again trianglelike (and flat) but with a rounded outer border, as shown for $p_{1}=p_{2}$ (see the text).

From Eq. (33) for $\beta_{2}=0$, it can be seen that the cone vertex will lie outside the ellipsoid whenever

$$
p_{2} \geqslant \frac{p_{1} \sin ^{2} \beta_{1}}{2\left(1-\cos \beta_{1} \cos \gamma\right)}
$$

(if $p_{1}<0$, the inequality should be inverted). The straight lines delimiting the cone end at the ellipsoid points $(x, y, z)$ satisfying $\frac{x\left(x-x_{v}\right)+y\left(y-y_{v}\right)}{b^{2}}+\frac{\left(z-z_{c}\right)\left(z-z_{v}\right)}{a^{2}}=0$. We also note that the entanglement of (19) is in this case driven just by $\left|\Psi_{1}\right\rangle$, with the partial transpose becoming nonpositive just for $p_{1} \sin \beta_{1}>p_{0} / d_{A}$, i.e., $p_{1}>\left(1-p_{2}\right) /\left(1+d_{A} \sin \beta_{1}\right)$.

$b$. Triangles and segments. If $\left|\Psi_{1}\right\rangle$ also becomes separable $\left(\beta_{1}=0\right)$, the first ellipsoid reduces to a segment, linking the origin with $\boldsymbol{r}_{1}=p_{1}(0,0,1) /\left(p_{1}+p_{0} / d_{A}\right)$. The ensuing convex hull of both segments leads to a two-dimensional triangle if they are noncollinear, i.e., $\gamma \neq 0$ in (36), with vertices at the origin, $\boldsymbol{r}_{1}$ and $\boldsymbol{r}_{2}=\boldsymbol{r}_{v}$, as shown in Fig. 5(a). This result holds whenever the maximally mixed state has nonzero weight, i.e., $p_{1}+p_{2}<1$ and $d_{A} \geqslant 3$.

On the other hand, if $p_{1}+p_{2}=1$, the previous segments reduce to the points $\boldsymbol{r}_{1}$ and $\boldsymbol{r}_{2}$ on the Bloch sphere and their convex hull becomes just the segment between them, leading to a needle-type state like that in Fig. 5(b). The final set does not depend on the ratio $p_{2} / p_{1}$, as long as both probabilities are nonzero, and holds in this case for any $d_{A} \geqslant 2$.

\section{Mixture of two separable pure states}

Needle shapes actually emerge from any mixture of two pure product states, i.e.,

$$
\begin{aligned}
\rho_{A B} & =p_{1}\left|\Psi_{1}\right\rangle\left\langle\Psi_{1}\left|+p_{2}\right| \Psi_{2}\right\rangle\left\langle\Psi_{2}\right|, \\
\left|\Psi_{i}\right\rangle & =\left|\psi_{i}^{A}\right\rangle\left|\psi_{i}^{B}\right\rangle, \quad i=1,2,
\end{aligned}
$$

where both local states $\left|\psi_{i}^{A}\right\rangle$ and $\left|\psi_{i}^{B}\right\rangle, i=1,2$, are now completely arbitrary (and $p_{1}+p_{2}=1$ ). The mixture (38) can be conveniently rewritten as

$$
\rho_{A B}=p_{+}\left|\theta_{A} \theta_{B}\right\rangle\left\langle\theta_{A} \theta_{B}\left|+p_{-}\right|-\theta_{A}-\theta_{B}\right\rangle\left\langle-\theta_{A}-\theta_{B}\right|,
$$

where $p_{+(-)}=p_{1(2)}$ and $\left| \pm \theta_{S}\right\rangle=e^{\mp i \phi_{S} / 2}\left|\psi_{1,2}^{S}\right\rangle$ for $S=A, B$, with $\phi_{S}$ and $\theta_{S} \in[0, \pi / 2]$ determined by

$$
\left\langle\psi_{2}^{S} \mid \psi_{1}^{S}\right\rangle=e^{i \phi_{S}} \cos \theta_{S}, \quad S=A, B
$$

such that $\left\langle-\theta_{S} \mid \theta_{S}\right\rangle=\cos \theta_{S}$. In this way, $\left| \pm \theta_{S}\right\rangle=\cos \frac{\theta_{S}}{2}\left|0_{S}\right\rangle \pm$ $\sin \frac{\theta_{S}}{2}\left|1_{S}\right\rangle$ can be seen as qubit states rotated an angle $\pm \theta_{S}$ from states $\left|0_{S}\right\rangle$ around the $y$ axis, with

$$
\left|0_{S}\right\rangle=\frac{\left|\theta_{S}\right\rangle+\left|-\theta_{S}\right\rangle}{2 \cos \frac{\theta_{S}}{2}}, \quad\left|1_{S}\right\rangle=\frac{\left|\theta_{S}\right\rangle-\left|-\theta_{S}\right\rangle}{2 \sin \frac{\theta_{S}}{2}}
$$

orthonormal states.

A general pure state $\left|\boldsymbol{k}_{A}\right\rangle$ of qudit $A$ can now be written as in Eq. (9), with $\left|\boldsymbol{k}_{A}^{\|}\right\rangle$of the form (12). The ensuing conditional state of $B$,

$$
\rho_{B / \boldsymbol{k}}=p_{+}^{\prime}(\boldsymbol{k})\left|\theta_{B}\right\rangle\left\langle\theta_{B}\left|+p_{-}^{\prime}(\boldsymbol{k})\right|-\theta_{B}\right\rangle\left\langle-\theta_{B}\right|,
$$

has the same form as the original state $\rho_{B}=\operatorname{Tr}_{A} \rho_{A B}=$ $p_{+}\left|\theta_{B}\right\rangle\left\langle\theta_{B}\left|+p_{-}\right|-\theta_{B}\right\rangle\left\langle-\theta_{B}\right|$, but with modified weights

$$
p_{ \pm}^{\prime}(\boldsymbol{k})=\frac{p_{ \pm}\left|\left\langle\boldsymbol{k}_{A}^{\|} \mid \pm \theta_{A}\right\rangle\right|^{2}}{p_{+}\left|\left\langle\boldsymbol{k}_{A}^{\|} \mid \theta_{A}\right\rangle\right|^{2}+p_{-}\left|\left\langle\boldsymbol{k}_{A}^{\|} \mid-\theta_{A}\right\rangle\right|^{2}},
$$

which cover all values between 0 and 1 as $\left|\boldsymbol{k}_{A}^{\|}\right\rangle$is varied [since $p_{ \pm}^{\prime}(\boldsymbol{k})=0$ when $\left|\boldsymbol{k}_{A}^{\|}\right\rangle$is orthogonal to $\left| \pm \theta_{A}\right\rangle$ ]. Consequently, the set of postmeasurement Bloch vectors $\boldsymbol{r}_{B / \boldsymbol{k}}=\sum_{\nu= \pm} p_{ \pm}^{\prime}(\boldsymbol{k}) \boldsymbol{r}_{B \pm}$, with $\boldsymbol{r}_{B \pm}=\left\langle \pm \theta_{B}|\boldsymbol{\sigma}| \pm \theta_{B}\right\rangle=$ ( $\pm \sin \theta_{B}, 0, \cos \theta_{B}$ ), is always the full segment joining the points $\boldsymbol{r}_{B \pm}$ located on the Bloch sphere surface, as shown in Fig. 5(b).

This result holds for any qudit dimension $d_{A} \geqslant 2$ and is then similar to that for a two-qubit system in a similar state (the needle is in fact a limiting case of an ellipsoid), since the local support at $A$ of the state (38) is two dimensional. Differences with the two-qubit case arise only when the support of $\rho_{A B}$ involves a qudit subspace of higher dimension, as was shown in the examples of Figs. 1, 3, 4, and 5(a).

For example, if we now mix the states (38) and (39) with the maximally mixed state, as in Eq. (19), $\rho_{B / \boldsymbol{k}}$ will be a mixture of the state (42) with the maximally mixed state $\mathbb{1}_{B} / 2$. Using (9)-(12), the ensuing conditional Bloch vector becomes $\boldsymbol{r}_{B / \boldsymbol{k}}=q \sum_{\nu= \pm} \tilde{p}_{ \pm}(\boldsymbol{k}) \boldsymbol{r}_{B \pm}, \quad$ with $\quad \tilde{p}_{ \pm}(\boldsymbol{k})=p_{ \pm}^{\prime}(\boldsymbol{k}) /\left(q+p_{0} /\right.$ $\left.\left[d_{A} \sum_{\nu= \pm}\left|\left\langle\boldsymbol{k}_{A}^{\|} \mid \nu \theta_{A}\right\rangle\right|^{2}\right]\right)$ and $p_{0}=1-p_{+}-p_{-}$. In the twoqubit case $d_{A}=2, q=1$ and the set of conditional vectors will form a flat filled ellipse (pancake shape, the limiting case of an ellipsoid surface) in the $x z$ plane. However, for $d_{A} \geqslant 3$ qudit states orthogonal to both $\left| \pm \theta_{A}\right\rangle$ exist and hence $q \in[0,1]$, implying that such a set will become the convex 
mixture of a similar flat filled ellipse with the origin. It will lead to a flat shape like that shown in Fig. 5(c), i.e., trianglelike but with a rounded upper border, obtained for $p_{+}=p_{-}=0.3, d_{A}=4, \theta_{A}=\pi / 4$, and $\theta_{B}=\pi / 6$.

\section{MINIMUM CONDITIONAL ENTROPY}

\section{A. Measurement-determined conditional entropy}

The concept of a measurement-determined quantum conditional entropy of a bipartite system was originally introduced in connection with the quantum discord [2,3,19-22]. It is a measure of the average conditional mixedness of the unmeasured subsystem $B$ after a local measurement at $A$, and its minimum over all local measurements determines the quantum discord. Such minimization is in general a difficult problem, shown to be NP-complete [46]. The concept was later extended to generalized entropic forms [14,18], which can enable a simpler evaluation and an analytic determination of the minimum in some cases. Here we will discuss the generalized conditional entropy in the states considered in the preceding section, providing analytic results for its minimizing measurement and its geometric picture. Such a measurement is also interesting in itself, since it maximizes the average amount of information on $B$ that can be gained through measurements at $A$.

Given a local measurement at $A$ determined by measurement operators $M_{j}=M_{j}^{A} \otimes \mathbb{1}_{B}$, satisfying $\sum_{j} M_{j}^{\dagger} M_{j}=\mathbb{1}_{A B}$, the generalized measurement-dependent conditional entropy $S_{f}\left(B \mid A_{M}\right)$ is defined as $[14,18]$

$$
S_{f}\left(B \mid A_{M}\right)=\sum_{j} p_{j} S_{f}\left(\rho_{B / j}\right),
$$

where $p_{j}=\operatorname{Tr}\left[\rho_{A B} M_{j}^{\dagger} M_{j}\right]$ is the probability of outcome $j$ and $\rho_{B / j}=p_{j}^{-1} \operatorname{Tr}_{A}\left[\rho_{A B} M_{j}^{\dagger} M_{j}\right]$ the conditional state of $B$ after this outcome. Here

$$
S_{f}(\rho)=\operatorname{Tr} f(\rho)
$$

is a trace form entropy [47,48], with $f:[0,1] \rightarrow \mathbb{R}$ a smooth strictly concave function satisfying $f(0)=f(1)=0$ [implying $S_{f}(\rho)$ concave and $S_{f}(\rho) \geqslant 0$, with $S_{f}(\rho)=0$ if and only if $\rho$ is a pure state]. For $f(p)=-p \log _{2} p$, Eq. (45) becomes the von Neumann entropy $S(\rho)=-\operatorname{Tr} \rho \log _{2} \rho$, while for $f(p)=2 p(1-p)$, it becomes the linear entropy

$$
S_{2}(\rho)=2\left(1-\operatorname{Tr} \rho^{2}\right),
$$

also known as quadratic entropy and coincident with the $q=2$ Tsallis entropy [49], obtained for $f(p) \propto \frac{p-p^{q}}{q-1}$.

Since $\rho_{B}=\sum_{j} p_{j} \rho_{B / j}$, concavity of $S_{f}$ directly implies $S_{f}(B) \equiv S_{f}\left(\rho_{B}\right) \geqslant \sum_{j} p_{j} S_{f}\left(\rho_{B / j}\right)=S_{f}\left(A \mid B_{M}\right)$, with equality if and only if $\rho_{B / j}=\rho_{B} \forall j$ with $p_{j}>0$. Thus, the generalized conditional entropy is never greater than the corresponding marginal entropy.

Its minimum over all local measurements at $A$,

$$
S_{f}(B \mid A)=\min _{M} S_{f}\left(B \mid A_{M}\right),
$$

depends just on $\rho_{A B}$, with $\Delta S_{f}(B \mid A)=S_{f}(B)-S_{f}(B \mid A)$ a non-negative quantity that measures the maximum average conditional information gain about $B$, as measured by $S_{f}$, that can be obtained through a measurement at $A$. The $\Delta S_{f}(B \mid A)$ vanishes if and only if $\rho_{A B}$ is a product state. The minimum (47) also represents the generalized entanglement of formation [50] $E_{f}(B, C)$ of the system of $B$ and $C$, where $C$ is a third system purifying the whole system $[18,23]$. In the case of the von Neumann entropy, the minimum $S(B \mid A)$ determines the quantum discord through $[19,20] D(B \mid A)=S(B \mid A)-$ $\tilde{S}(B \mid A)$, where $\tilde{S}(B \mid A)=S\left(\rho_{A B}\right)-S\left(\rho_{A}\right)$ is the standard quantum conditional entropy [47]. While the latter is negative in any pure entangled state, the minimum (47) is obviously always non-negative, vanishing in any pure state [14].

\section{B. Minimizing measurement}

The minimum (47) can be always reached for measurements based on rank-1 operators $M_{j}=\sqrt{r_{j}} \Pi_{\boldsymbol{k}_{j}}^{A}[2,3]$, of the type considered in Sec. II, a result which holds for any $S_{f}$ $[14,18]$. It is then sufficient to consider

$$
S_{f}\left(B \mid A_{M}\right)=\sum_{j} r_{j} p_{\boldsymbol{k}_{j}} S_{f}\left(\rho_{B / \boldsymbol{k}_{j}}\right),
$$

with $\rho_{B / k_{j}}$ given by Eq. (6) and projectors $\Pi_{k}^{A}$ within the local support at $A$ of $\rho_{A B}$, as discussed below Eq. (9).

For a mixture of a single pure state $|\Psi\rangle$ with the maximally mixed state (10), the minimum (47) is reached, for any $S_{f}$, for a projective measurement on the local Schmidt basis $\left\{\left|k_{A}\right\rangle\right\}$ determined by the state $|\Psi\rangle$ [18]. In the Appendix it is shown that this result can be extended to any mixture of the form (19), where the local supports at $A$ of the states $\left|\Psi_{i}\right\rangle$ are orthogonal and hence compatible with a unique local Schmidt basis:

$$
\begin{aligned}
\rho_{A B} & =\sum_{i=1}^{n} p_{i}\left|\Psi_{i}\right\rangle\left\langle\Psi_{i}\right|+p_{0} \frac{\mathbb{1}_{A B}}{d_{A} d_{B}}, \\
\left|\Psi_{i}\right\rangle & =\sum_{k} \sqrt{q_{i k}}\left|k_{A}\right\rangle\left|k_{i B}\right\rangle,
\end{aligned}
$$

where $p_{0}=1-\sum_{i=1}^{n} p_{i} \geqslant 0\left(p_{i} \geqslant 0\right)$ and $q_{i k}=\delta_{i, i_{k}} q_{k}\left(q_{k}>0\right)$ such that for each $k$ there is at most a single state $\left|\Psi_{i_{k}}\right\rangle$ with finite overlap with $\left|k_{A}\right\rangle$. Hence, for a measurement in the basis $\left\{\left|k_{A}\right\rangle\right\}$

$$
\rho_{B / k}=\frac{1}{p_{k}^{A}}\left(p_{i_{k}} q_{k}\left|k_{i_{k} B}\right\rangle\left\langle k_{i_{k} B}\right|+p_{0} \frac{\mathbb{1}_{B}}{d_{A} d_{B}}\right),
$$

where $p_{k}^{A}=p_{i_{k}} q_{k}+p_{0} / d_{A}$. The ensuing minimum conditional entropy is then

$$
\begin{aligned}
S_{f}(B \mid A)= & \sum_{k} p_{k}^{A}\left[f\left(\frac{d_{A} d_{B} p_{i_{k}} q_{k}+p_{0}}{d_{A} d_{B} p_{k}^{A}}\right)\right. \\
& \left.+\left(d_{B}-1\right) f\left(\frac{p_{0}}{d_{A} d_{B} p_{k}^{A}}\right)\right] .
\end{aligned}
$$

In the von Neumann case, this expression enables a direct evaluation of the quantum discord $D(B \mid A)$.

Consequently, for the states of Secs. II B and II C, the postmeasurement states of the qubit determined by the minimizing measurement at the qudit have a clear geometric picture: In the states (10) and (11), the minimum is obtained for a projective measurement in a basis containing the states $\left|0_{A}\right\rangle$ and $\left|1_{A}\right\rangle$, i.e., 
$\alpha=0$ and $\pi$ in (12), with $q=1$. The associated conditional qubit Bloch vectors lie at the ellipsoid extrema along the major (z) axis. Similarly, for the states (19) the minimizing measurement basis should contain in addition the states $\left|2_{A}\right\rangle$ and $\left|3_{A}\right\rangle$, and the ensuing conditional qubit vectors lie at the ellipsoids major axis extrema. For $\beta_{2}=0$ (ice-cream shapes), this leads to the ellipsoid extrema and the cone vertex, i.e., $q^{\prime}=1 ; q=1 ; \alpha_{1}=0, \pi$; and $q=0 ; \alpha_{2}=0$ in (22) and (23), while for a triangle shape, it leads to the triangle vertices.

\section{Quadratic conditional entropy}

For more general states $\rho_{A B}$, the problem of determining the minimizing measurement is in general hard [46]. It is then convenient to consider the quadratic conditional entropy derived from (46) [14], which is determined by the state purity and hence does not require the knowledge of its eigenvalues and which can in principle be accessed experimentally without the need of a full state tomography [51]. First, by means of Eqs. (1) and (3), the quadratic marginal entropy can be evaluated explicitly as

$$
S_{2}\left(\rho_{S}\right)=\frac{2}{d_{S}}\left(d_{S}-1-\left|\boldsymbol{r}_{S}\right|^{2}\right), \quad S=A, B,
$$

which shows that $\left|\boldsymbol{r}_{S}\right|^{2} \leqslant d_{S}-1$, with equality if and only if $\rho_{S}$ is pure. The corresponding conditional entropy (48) can also be explicitly determined using Eqs. (6) and (7) [14],

$$
S_{2}\left(B \mid A_{M}\right)=S_{2}\left(\rho_{B}\right)-\Delta S_{2}\left(B \mid A_{M}\right),
$$

where

$$
\Delta S_{2}\left(B \mid A_{M}\right)=\frac{1}{d_{B}} \sum_{j} r_{j} \frac{\left|C^{T} \boldsymbol{k}_{j}\right|^{2}}{1+\boldsymbol{r}_{A} \cdot \boldsymbol{k}_{j}}
$$

is a non-negative quantity representing an information gain. Here $C$ is the correlation tensor (4) and $\boldsymbol{r}_{A}=\left\langle\boldsymbol{\sigma}_{A}\right\rangle$.

In particular, if the local support at $A$ of state $\rho_{A B}$ involves just two pure states $\left|0_{A}\right\rangle$ and $\left|1_{A}\right\rangle$, we may directly consider projectors within this subspace $\mathcal{S}$ and use effective Pauli operators at system $A$. For a standard projective measurement based on the orthogonal states $\left| \pm \boldsymbol{k}_{A}\right\rangle$, with $\left\langle \pm \boldsymbol{k}_{A}|\boldsymbol{\sigma}| \pm \boldsymbol{k}_{A}\right\rangle=$ $\pm \boldsymbol{k}$, Eq. (55) reduces to [14]

$$
\Delta S_{2}\left(B \mid \boldsymbol{k}_{A}\right)=\frac{2}{d_{B}} \frac{\left|C^{T} \boldsymbol{k}\right|^{2}}{1-\left(\boldsymbol{r}_{A} \cdot \boldsymbol{k}\right)^{2}}=\frac{2}{d_{B}} \frac{\boldsymbol{k}^{T} C C^{T} \boldsymbol{k}}{\boldsymbol{k}^{T} N_{A} \boldsymbol{k}},
$$

where $N_{A}=\mathbb{1}-\boldsymbol{r}_{A} \boldsymbol{r}_{A}^{T}$. Maximization of (56) [equivalent to minimization of (54)] over these measurements is then achieved by solving the weighted eigenvalue problem

$$
C C^{T} \boldsymbol{k}=\lambda N_{A} \boldsymbol{k}
$$

and selecting the largest eigenvalue $\lambda_{\max }$, with the optimizing measurement determined by the corresponding eigenvector $\boldsymbol{k}$ (i.e., it is an effective spin-1/2 measurement in $\mathcal{S}$ along direction $\boldsymbol{k}$ ). This leads to

$$
\min _{\boldsymbol{k}} S_{2}\left(B \mid \boldsymbol{k}_{A}\right)=S_{2}\left(\rho_{B}\right)-\frac{2}{d_{B}} \lambda_{\max } .
$$

More general POVM measurements based on an arbitrary set $\left\{r_{j} \Pi_{\boldsymbol{k}_{j}}^{A}\right\}$ do not improve previous minimum [14].
Hence, for these states the linear entropy allows a direct analytic evaluation of the associated minimum conditional entropy and its minimizing measurement. As a check, for a two-qubit state $\rho_{A B}=p|\Psi\rangle\langle\Psi|+(1-p) \mathbb{1} / 4$, with $|\Psi\rangle$ of the form (11), $\boldsymbol{r}_{A(B)}=(0,0, p \cos \beta)$ and $C_{\mu \nu}=\delta_{\mu \nu} C_{\mu \mu}$, with $C_{x x}=-C_{y y}=p \sin \beta$ and $C_{z z}=p\left(1-p \cos ^{2} \beta\right)$. It is then verified that the largest eigenvalue of Eq. (57) is $\lambda_{z}=C_{z z}^{2} /\left(1-p^{2} \cos ^{2} \beta\right)\left(\lambda_{z}>\lambda_{x}=\lambda_{y}=p^{2} \sin ^{2} \beta\right)$, associated with the eigenvector $\boldsymbol{k}_{z}=(0,0,1)$, implying measurement in the Schmidt basis $\left\{\left|0_{A}\right\rangle,\left|1_{A}\right\rangle\right\}$. Equation (58) then coincides with (52) for $f(p)=2 p(1-p)$.

\section{Case of rank-2 separable states}

Previous expressions enable us to determine the minimum conditional entropy in the state (39) and the associated minimizing measurement. Of course, if $\theta_{A}=\pi / 2$, states $\left| \pm \theta_{A}\right\rangle$ are orthogonal and a projective measurement on this basis, i.e., a spin measurement along the $x$ axis in the qubit picture, provides the minimum $\left[S_{f}(A \mid B)=0\right]$.

For general $\theta$, we obtain $C_{\mu \nu}=\delta_{\mu \nu} \delta_{\mu, x} C_{x x}$, with $C_{x x}=4 p_{+} p_{-} \sin \theta_{A} \sin \theta_{B}, \quad$ while for $S=A, B, \quad \boldsymbol{r}_{S}=$ $\left(\left(p_{+}-p_{-}\right) \sin \theta_{S}, 0, \cos \theta_{S}\right)$. The matrix $N_{A}$ in (57) is then nondiagonal if $p_{+} \neq p_{-}$and $\theta_{A} \in(0, \pi / 2)$, and Eq. (57) leads to $\boldsymbol{k}=(\sin \phi, 0, \cos \phi)$, with

$$
\tan \phi=\frac{\tan \theta_{A}}{p_{+}-p_{-}}
$$

if $p_{+} \geqslant p_{-}$(i.e., $p_{+} \geqslant 1 / 2$ ). This entails a spinlike measurement at the effective qubit along a direction $\boldsymbol{k}$ forming an angle $\phi$ with the $z$ axis such that

$$
\left|\boldsymbol{k}_{A}\right\rangle=\left|\phi_{A}\right\rangle, \quad\left|-\boldsymbol{k}_{A}\right\rangle=\left|(\phi+\pi)_{A}\right\rangle .
$$

The meaning of the minimizing angle (59) is that the ensuing entropies $S_{2}\left(\rho_{B / \pm \boldsymbol{k}}\right)$ are equal, i.e., the vectors $\boldsymbol{r}_{B / \pm \boldsymbol{k}}$ both have the same length:

$$
\boldsymbol{r}_{B / \pm \boldsymbol{k}}=\left[ \pm \sin \theta_{B} \sqrt{\left(p_{+}-p_{-}\right)^{2} \cos ^{2} \theta_{A}+\sin ^{2} \theta_{A}}, 0, \cos \theta_{B}\right]
$$

These vectors are not at the edges $\left( \pm \sin \theta_{B}, 0, \cos \theta_{B}\right)$ of the segment (except when the states $\left| \pm \theta_{A}\right\rangle$ are orthogonal, i.e., $\theta_{A}=\pi / 2$ ), but rather at inner symmetric points with respect to the $z$ axis. The ensuing minimum conditional entropy (58) is just

$$
S_{2}(B \mid A)=1-\left|\boldsymbol{r}_{B / \pm \boldsymbol{k}}\right|^{2}=4 p_{+} p_{-} \cos ^{2} \theta_{A} \sin ^{2} \theta_{B} .
$$

It vanishes in the trivial cases $\theta_{A}=\pi / 2$ or $\theta_{B}=0$.

In the equally weighted case $p_{+}=p_{-}=1 / 2$, Eq. (59) leads to $\phi=\pi / 2$ for any $\theta_{A}$, i.e., to a spin measurement along the $x$ axis (states $\left| \pm \boldsymbol{k}_{A}\right\rangle=\frac{\left|0_{A}\right\rangle \pm\left|1_{A}\right\rangle}{\sqrt{2}}$ ), in agreement with the fact that $N_{A}$ becomes diagonal and the only nonzero correlation is $C_{x x}$. The solution (59) also can actually be obtained in this way, by considering (39) as an equally weighted mixture of unnormalized states $\sqrt{p_{ \pm}}\left| \pm \theta_{A}, \pm \theta_{B}\right\rangle$. The normalized (but nonorthogonal) states $\left|0_{A}^{\prime}\right\rangle$ and $\left|1_{A}^{\prime}\right\rangle$ associated with the latter are $\begin{aligned} & \left|0_{A}^{\prime}\right\rangle \\ & \left|1_{A}^{\prime}\right\rangle\end{aligned}=\frac{\sqrt{p_{+}}\left|\theta_{A}\right\rangle \pm \sqrt{p_{-}}\left|-\theta_{A}\right\rangle}{\sqrt{1 \pm 2 \sqrt{p_{+} p_{-}} \cos \theta_{A}}}$, and the ensuing normalized orthogonal states along $x^{\prime},\left| \pm \boldsymbol{k}_{A}\right\rangle=\frac{\left|0_{A}^{\prime}\right\rangle \pm\left|1_{A}^{\prime}\right\rangle}{\sqrt{2\left(1 \pm\left\langle 1_{A}^{\prime} \mid 0_{A}^{\prime}\right\rangle\right)}}$, are precisely the states (60) determined by Eq. (59). 


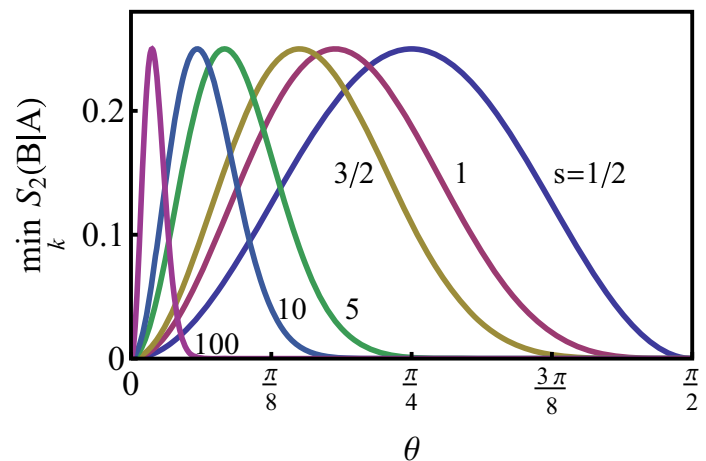

FIG. 6. Minimum conditional entropy (65) as a function of $\theta$ for the indicated values of the spin $s$ in the state (63).

A remarkable feature is that Eq. (59) determines the minimizing measurement for any conditional entropy $S_{f}\left(B \mid A_{M}\right)$ for which the entropy $S_{f}(\rho)=f(p)+f(1-p)$ of a single-qubit state $\rho$ is a convex increasing function of $\sqrt{S_{2}(\rho)}=2 \sqrt{p(1-p)}\left[S_{f}(\rho)=F\left(\sqrt{S_{2}(\rho)}\right)\right.$, with $F(x)=$ $\left.\sum_{v= \pm} f\left(\frac{1+v \sqrt{1-x^{2}}}{2}\right)\right]$. The reason is that for these states the system $C$ purifying the whole system is also a qubit, and hence for these entropies the entanglement of formation $E_{f}(B, C)=$ $S_{f}(B \mid A)$ is determined by the concurrence [42], which is just $\sqrt{E_{2}(B, C)}=\sqrt{S_{2}(B \mid A)}$. Thus, $S_{f}(B \mid A)=E_{f}(B, C)=$ $F\left(\sqrt{E_{2}(B, C)}\right)=F\left(\sqrt{S_{2}(B \mid A)}\right)$. In addition, since the $S_{2}$ minimizing measurement leads to coincident postmeasurement entropies $S_{f}\left(\rho_{B / \pm k}\right)=F\left(\sqrt{S_{2}(B \mid A)}\right)$, it also minimizes $S_{f}\left(A \mid B_{M}\right)$ for such entropies. Convexity of $F$ holds, in particular, for the Tsallis entropies with $\frac{5-\sqrt{13}}{2} \leqslant q \leqslant \frac{5+\sqrt{13}}{2}$ [52], including the von Neumann entropy (recovered for $q \rightarrow 1$ ). Hence, the present results also enable a direct evaluation of the quantum discord for these states and are in agreement with those of [7].

\section{E. Mixture of aligned spin-s states}

As an application of the previous result, we finally consider the case of two actual spins $s$ in a mixture of two maximally aligned states $|\nearrow \nearrow\rangle=\left|\theta_{s} \theta_{s}\right\rangle$ and $|\nwarrow \nwarrow\rangle=\left|-\theta_{s}-\theta_{s}\right\rangle$, in directions forming angles $\pm \theta$ with the $z$ axis. These states arise, for instance, as exact reduced pair states in the ground state of $X Y$ and $X Y Z$ spin chains in an applied transverse field along $z$, in the immediate vicinity of the factorizing field $[53,54]$. Their joint state takes the form (39), i.e.,

$$
\rho_{A B}=p_{+}\left|\theta_{s} \theta_{s}\right\rangle\left\langle\theta_{s} \theta_{s}\left|+p_{-}\right|-\theta_{s}-\theta_{s}\right\rangle\left\langle-\theta_{s}-\theta_{s}\right|,
$$

with $p_{ \pm}=1 / 2$ and $\left| \pm \theta_{s}\right\rangle=e^{\mp i \theta S_{y}}|m=s\rangle$ given by

$$
\left| \pm \theta_{s}\right\rangle=\sum_{m=-s}^{s} \sqrt{\left(\begin{array}{c}
2 s \\
m+s
\end{array}\right)} \cos ^{s+m}\left(\frac{\theta}{2}\right) \sin ^{s-m}\left(\frac{ \pm \theta}{2}\right)|m\rangle .
$$

Since $\left\langle-\theta_{s} \mid \theta_{s}\right\rangle=\cos ^{2 s} \theta_{s}$, these states correspond to an effective qubit angle $\theta_{A}=\theta_{B}$ in (39), with $\cos \theta_{A}=\cos ^{2 s} \theta$. Hence, according to Eq. (62), the minimum $S_{2}$ conditional entropy is

$$
S_{2}(B \mid A)=4 p_{+} p_{-} \cos ^{4 s} \theta\left(1-\cos ^{4 s} \theta\right) .
$$

In Fig. 6 we depict $S_{2}(B \mid A)$ vs $\theta$ for different values of the spin $s$ in the equally probable case $p_{ \pm}=1 / 2$. Its maximum is $s$

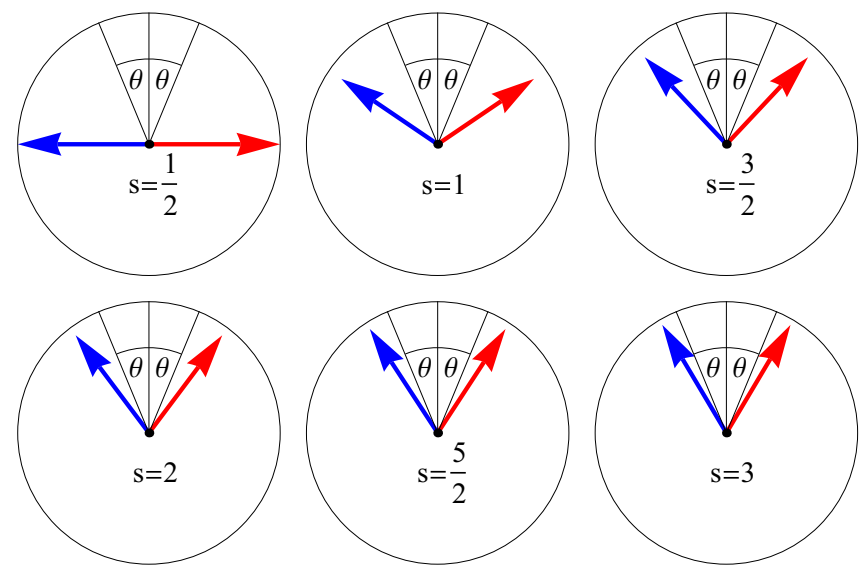

FIG. 7. Scaled averages (66) of the spin operator $S$ in the orthogonal states $\left| \pm \boldsymbol{k}_{A}\right\rangle$ which minimize the $S_{2}$ conditional entropy, for the joint state (63). As the spin $s$ increases they approach the $\pm \theta$ directions.

independent but is reached at $\theta=\theta_{m}=\arccos \left(2^{-1 / 4 s}\right)$, which vanishes for large $s\left(\theta_{m} \approx \sqrt{\frac{\ln 2}{2 s}}\right.$ for $\left.s \gg 1\right)$. The minimum conditional entropy $S_{2}(B \mid A)$ becomes then very small for $\theta>$ $\theta_{m}$ and large $s$, as states $\left| \pm \theta_{s}\right\rangle$ become almost orthogonal.

On the other hand, the local measurement minimizing the conditional entropy is that in an orthogonal basis of states containing the states (60), where $\left|\phi_{A}\right\rangle=\cos \frac{\phi}{2}\left|0_{A}\right\rangle+$ $\sin \frac{\phi}{2}\left|1_{A}\right\rangle$ with [Eq. (41)] $\left.\left.\right|_{1_{A}} ^{0_{A}}\right\rangle=\frac{\left|\theta_{s}\right\rangle \pm\left|-\theta_{s}\right\rangle}{\sqrt{2\left(1 \pm \cos ^{2} \theta\right)}}$. For $s \geqslant 1$, such a measurement is not a spin measurement, in the sense that it does not correspond to the measurement of the spin at a certain direction $\boldsymbol{n}$ : In the latter, the spin has collinear integer values $\left\langle m_{\boldsymbol{n}}|\boldsymbol{S}| m_{\boldsymbol{n}}\right\rangle=m \boldsymbol{n}$, with $m=-s, \ldots, s$, whereas in the minimizing states (60), it takes nonparallel and noninteger average values, as occurs for general projective measurements (see [55]). For $p_{ \pm}=1 / 2$ and $\phi=\pi / 2$ we obtain

$$
\begin{aligned}
& \left\langle \pm \boldsymbol{k}_{A}|\boldsymbol{S}| \pm \boldsymbol{k}_{A}\right\rangle / s \\
& \quad=\left( \pm \frac{\sin \theta}{\sqrt{1-\cos ^{4 s} \theta}}, 0, \frac{\cos \theta\left(1-\cos ^{4 s-2} \theta\right)}{1-\cos ^{4 s} \theta}\right) .
\end{aligned}
$$

As seen in Fig. 7, while for $s=1 / 2$ the vectors $\langle\boldsymbol{s}\rangle_{ \pm}=$ $\left\langle \pm \boldsymbol{k}_{A}|\boldsymbol{S}| \pm \boldsymbol{k}_{A}\right\rangle / s$ point along the $x$ axis, indicating a spin measurement along the $x$ axis, for $s \geqslant 1$ they are noncollinear and approach, for large $s$, the $\pm \theta$ directions, i.e., $\langle\boldsymbol{s}\rangle_{ \pm} \approx$ $\left\langle \pm \theta_{s}|\boldsymbol{S}| \pm \theta_{s}\right\rangle / s=( \pm \sin \theta, 0, \cos \theta)$, coinciding with the latter for $s \rightarrow \infty$. This result is to be expected as in this limit the states $\left| \pm \theta_{s}\right\rangle$ become orthogonal. In the case of spin $s=1$, the averages (66) imply that the minimizing measurement is a Y-type projective measurement, following the terminology of [55], based on the states $\left| \pm \boldsymbol{k}_{A}\right\rangle$ and a third state orthogonal to the latter.

\section{CONCLUSION}

We have shown that in correlated mixed states of quditqubit systems, the set of all conditional qubit states after a general local measurement at the qudit based on rank-1 projectors may exhibit geometries which are more complex 
than a single ellipsoid. While a single solid ellipsoid, with the origin as one of its foci, is obtained for a state which is the mixture of a pure entangled state with the maximally mixed state, for more general mixtures of the form (19) such set becomes the convex hull of different solid ellipsoids, leading to shapes like that of Fig. 3. These shapes may become conelike or flat trianglelike when one or more of the pure states of the mixture are separable, as shown in Figs. 4 and 5.

We have also analyzed the corresponding measurementdependent conditional entropy and its minimizing measurement for the previous states. For a mixture of a single pure state with the maximally mixed state, such a measurement is that on the pure state Schmidt basis and is universal, in the sense of minimizing any entropy of the form (45). We have shown that this result can be extended to any mixture (19) where the local supports of the states $\left|\Psi_{i}\right\rangle$ at the qudit side are orthogonal, leading to a clear geometric interpretation of the minimizing measurement in the set of postmeasurement qubit states. These minimizing measurements maximize the average conditional information gain about $B$ and enable the determination of the quantum discord. We also examined the case of rank-2 separable states (38) and (39), determining the minimizing measurement analytically for a wide class of entropies through the linear entropy. As an application, the minimizing measurement for a mixture of maximally aligned two-spin states was determined for general spin $s$ and shown not to correspond to a standard spin measurement for any spin $s \geqslant 1$. Present results are suitable for experimental verification through optical means.

\section{ACKNOWLEDGMENT}

The authors acknowledge support from CONICET (M.B., N.C., and N.G.) and CIC (R.R.) of Argentina.

\section{APPENDIX}

We prove here that the measurement at the qudit $A$ minimizing the general conditional entropy (44) for states of the form (49) and (50), where the states $\left|\Psi_{i}\right\rangle$ have orthogonal local supports at $A$, is on the local Schmidt basis.

Proof. For a local measurement based on rank-1 operators $\sqrt{r_{j}}\left|j_{A}\right\rangle\left\langle j_{A}\right|$, with $\sum_{j} r_{j}\left|j_{A}\right\rangle\left\langle j_{A}\right|=\mathbb{1}_{d_{A}}$, we have $\rho_{A B / j}=$ $\Pi_{j}^{A} \rho_{A B} \Pi_{j}^{A} / p_{j}^{A}$, where $\Pi_{j}^{A}=\left|j_{A}\right\rangle\left\langle j_{A}\right| \otimes \mathbb{1}_{d_{B}}$ and

$$
p_{j}^{A}=\sum_{i} p_{i} \tilde{q}_{i j}+p_{0} / d_{A},
$$

with $r_{j} p_{j}^{A}$ the probability of result $j$, while

$$
\tilde{q}_{i j}=\sum_{k} q_{i k}\left|\left\langle j_{A} \mid k_{A}\right\rangle\right|^{2},
$$

with $r_{j} \tilde{q}_{i j}$ the probability of result $j$ in the state $\left|\Psi_{i}\right\rangle$. The ensuing conditional state at $B$ is

$$
\rho_{B / j}=\frac{1}{p_{j}^{A}}\left[\sum_{i} p_{i} \tilde{q}_{i j}\left|j_{i B}\right\rangle\left\langle j_{i B}\right|+p_{0} \frac{\mathbb{1}_{d_{B}}}{d_{A} d_{B}}\right],
$$

where $\left|j_{i B}\right\rangle=\frac{1}{\sqrt{\tilde{q}_{i j}}} \sum_{k} \sqrt{q_{i k}}\left\langle j_{A} \mid k_{A}\right\rangle\left|k_{i B}\right\rangle$. In terms of the conditional states (51) obtained for a measurement in the local Schmidt basis, we may rewrite (A3) as

$$
\rho_{B / j}=\sum_{k} \frac{\left|\left\langle j_{A} \mid k_{A}\right\rangle\right|^{2} p_{k}^{A}}{p_{j}^{A}} U_{j k} \rho_{B / k} U_{j k}^{\dagger},
$$

where $U_{j k}$ is a unitary operator satisfying $U_{j k}\left|k_{i_{k} B}\right\rangle=\left|j_{i_{k} B}\right\rangle$ and $\sum_{k} \frac{\left|\left\langle j_{A} \mid k_{A}\right\rangle\right|^{2} p_{k}^{A}}{p_{j}^{A}}=1$. Therefore, concavity and completeness of the measurement operators imply

$$
\begin{aligned}
S_{f}\left(B \mid A_{M}\right) & =\sum_{j} r_{j} p_{j}^{A} S_{f}\left(\rho_{B / j}\right) \\
& \geqslant \sum_{j, k} r_{j}\left|\left\langle j_{A} \mid k_{A}\right\rangle\right|^{2} p_{k}^{A} S_{f}\left(\rho_{B / k}\right) \\
& =\sum_{k} p_{k}^{A} S_{f}\left(\rho_{B / k}\right)=S_{f}(B \mid A) .
\end{aligned}
$$

[1] M. A. Nielsen and I. L. Chuang, Quantum Computation and Quantum Information (Cambridge University Press, Cambridge, 2000).

[2] K. Modi, A. Brodutch, H. Cable, T. Paterek, and V. Vedral, Rev. Mod. Phys. 84, 1655 (2012).

[3] G. Adesso, T. R. Bromley, and M. Cianciaruso, J. Phys. A: Math. Theor. 49, 473001 (2016).

[4] A. Ferraro, L. Aolita, D. Cavalcanti, F. M. Cucchietti, and A. Acín, Phys. Rev. A 81, 052318 (2010).

[5] G. de Chiara and A. Sanpera, Rep. Prog. Phys. 81, 074002 (2018).

[6] F. Verstraete, A study of entanglement in quantum information theory, Ph.D. thesis, Katholieke Universiteit Leuven, 2002.

[7] M. Shi, F. Jiang, C. Sun, and J. Du, New J. Phys. 13, 073016 (2011); M. Shi, W. Yang, F. Jiang, and J. Du, J. Phys. A: Math. Theor. 44, 415304 (2011).
[8] M. Shi, C. Sun, F. Jiang, X. Yan, and J. Du, Phys. Rev. A 85, 064104 (2012).

[9] K. K. Sabapathy and R. Simon, arXiv:1311.0210.

[10] S. Jevtic, M. Pusey, D. Jennings, and T. Rudolph, Phys. Rev. Lett. 113, 020402 (2014).

[11] A. Milne, D. Jennings, S. Jevtic, and T. Rudolph, Phys. Rev. A 90, 024302 (2014).

[12] A. Milne, S. Jevtic, D. Jennings, H. Wiseman, and T. Rudolph, New J. Phys. 16, 083017 (2014).

[13] R. McCloskey, A. Ferraro, and M. Paternostro, Phys. Rev. A 95, 012320 (2017).

[14] N. Gigena and R. Rossignoli, Phys. Rev. A 90, 042318 (2014).

[15] X. Hu and H. Fan, Phys. Rev. A 91, 022301 (2015).

[16] S. Cheng, A. Milne, M. J. W. Hall, and H. M. Wiseman, Phys. Rev. A 94, 042105 (2016).

[17] C. Zhang et al., Phys. Rev. Lett. 122, 070402 (2019). 
[18] N. Gigena and R. Rossignoli, J. Phys. A: Math. Theor. 47, 015302 (2014).

[19] H. Ollivier and W. H. Zurek, Phys. Rev. Lett. 88, 017901 (2001).

[20] L. Henderson and V. Vedral, J. Phys. A: Math. Gen. 34, 6899 (2001).

[21] N. Canosa, M. Cerezo, N. Gigena, and R. Rossignoli, in Lectures on General Quantum Correlations and their Applications, edited by F. F. Fanchini, D. d. O. Soares Pinto, and G. Adesso (Springer, Berlin, 2017), p. 455.

[22] A. Bera, T. Das, D. Sadhukhan, S. S. Roy, A. Sen(De), and U. Sen, Rep. Prog. Phys. 81, 024001 (2018).

[23] M. Koashi and A. Winter, Phys. Rev. A 69, 022309 (2004).

[24] E. Schrödinger, Proc. Camb. Philos. Soc. 31, 555 (1935); 32, 446 (1936).

[25] H. M. Wiseman, S. J. Jones, and A. C. Doherty, Phys. Rev. Lett. 98, 140402 (2007).

[26] P. Skrzypczyk, M. Navascués, and D. Cavalcanti, Phys. Rev. Lett. 112, 180404 (2014).

[27] I. Kogias, A. R. Lee, S. Ragy, and G. Adesso, Phys. Rev. Lett. 114, 060403 (2015).

[28] R. Gallego and L. Aolita, Phys. Rev. X 5, 041008 (2015).

[29] D. Cavalcanti and P. Skrzypczyk, Rep. Prog. Phys. 80, 024001 (2017).

[30] T. Kriváchy, F. Fröwis, and N. Brunner, Phys. Rev. A 98, 062111 (2018).

[31] G. Barreto Lemos, J. O. de Almeida, S. P. Walborn, P. H. Souto Ribeiro, and M. Hor-Meyll, Phys. Rev. A 89, 042119 (2014).

[32] K. H. Kagalwala, G. Di Giuseppe, A. F. Abouraddy, and B. E. A. Saleh, Nat. Commun. 8, 739 (2017); A. F. Abouraddy, G. Di Giuseppe, T. M. Yarnall, M. C. Teich, and B. E. A. Saleh, Phys. Rev. A 86, 050303(R) (2012).

[33] D. Pabón, L. Rebón, S. Bordakevich, N. Gigena, A. Boette, C. Iemmi, R. Rossignoli, and S. Ledesma, Phys. Rev. A 99, 062333 (2019).

[34] L. Neves, G. Lima, J. G. A. Gómez, C. H. Monken, C. Saavedra, and S. Pádua, Phys. Rev. Lett. 94, 100501 (2005).

[35] M. N. O'Sullivan-Hale, I. A. Khan, R. W. Boyd, and J. C. Howell, Phys. Rev. Lett. 94, 220501 (2005).
[36] J. W. Pan, Z. B. Chen, C. Y. Lu, H. Weinfurter, A. Zeilinger, and M. Zukowski, Rev. Mod. Phys. 84, 777 (2012).

[37] G. Lima, L. Neves, R. Guzmán, E. S. Gómez, W. A. T. Nogueira, A. Delgado, A. Vargas, and C. Saavedra, Opt. Express 19, 3542 (2011).

[38] J. Cho, D. G. Angelakis, and S. Bose, Phys. Rev. A 78, 062338 (2008)

[39] C. Senko, P. Richerme, J. Smith, A. Lee, I. Cohen, A. Retzker, and C. Monroe, Phys. Rev. X 5, 021026 (2015).

[40] E. Moreno-Pineda, C. Godfrin, F. Balestro, W. Wernsdorfer, and M. Ruben, Chem. Soc. Rev. 47, 501 (2018).

[41] U. Fano, Rev. Mod. Phys. 55, 855 (1983).

[42] S. Hill and W. K. Wootters, Phys. Rev. Lett. 78, 5022 (1997); W. K. Wootters, ibid. 80, 2245 (1998).

[43] A. Peres, Phys. Rev. Lett. 77, 1413 (1996).

[44] M. Horodecki, P. Horodecki, and R. Horodecki, Phys. Lett. A 223, 1 (1996).

[45] G. Vidal and R. F. Werner, Phys. Rev. A 65, 032314 (2002); K. Zyczkowski, P. Horodecki, A. Sanpera, and M. Lewenstein, ibid. 58, 883 (1998).

[46] Y. Huang, Phys. Rev. A 88, 014302 (2013).

[47] A. Wehrl, Rev. Mod. Phys. 50, 221 (1978).

[48] N. Canosa and R. Rossignoli, Phys. Rev. Lett. 88, 170401 (2002); Phys. Lett. A 264, 148 (1999).

[49] C. Tsallis, J. Stat. Phys. 52, 479 (1988).

[50] C. H. Bennett, D. P. DiVincenzo, J. A. Smolin, and W. K. Wootters, Phys. Rev. A 54, 3824 (1996).

[51] H. Nakazato, T. Tanaka, K. Yuasa, G. Florio, and S. Pascazio, Phys. Rev. A 85, 042316 (2012); T. Tanaka, G. Kimura, and H. Nakazato, ibid. 87, 012303 (2013).

[52] R. Rossignoli, N. Canosa, and L. Ciliberti, Phys. Rev. A 82, 052342 (2010).

[53] R. Rossignoli, N. Canosa, and J. M. Matera, Phys. Rev. A 77, 052322 (2008); 80, 062325 (2009).

[54] N. Canosa, R. Rossignoli, and J. M. Matera, Phys. Rev. B 81, 054415 (2010); A. Boette, R. Rossignoli, N. Canosa, and J. M. Matera, ibid. 94, 214403 (2016).

[55] R. Rossignoli, J. M. Matera, and N. Canosa, Phys. Rev. A 86, 022104 (2012). 